\title{
Révision partielle de la loi fédérale du 18 mars 1994 sur l'assurance-maladie. Projet 2A Financement des hôpitaux et 2B Managed Care
}

\author{
Monsieur le Conseiller fédéral \\ Pascal Couchepin \\ Département de l'intérieur \\ 3003 Berne \\ Berne, le 12 juillet 2004 YG/rd \\ Monsieur le Conseiller fédéral, \\ Mesdames et Messieurs,
}

La Fédération des médecins suisses (FMH) vous remercie de l'avoir consultée sur les objets susmentionnés.

\section{Projet 2A Financement hospitalier}

Si le projet dual fixe a le mérite de la transparence, il n'est pas sans poser un certain nombre de problèmes sur le plan social, de la répartition des tâches entre la LAMal et la LCA, ainsi que sur celui de la qualité des prestations.

Il aboutit fondamentalement à une modification de la répartition des charges au bout du compte aux dépens de l'assurance sociale et en faveur de l'assurance privée. Cette situation est pour le moins paradoxale au vu des difficultés rencontrées par nombre de ménages et d'assurés, puisqu'elle se soldera par une augmentation des primes, certes modérée, mais une augmentation tout de même. L'évolution des prestations, constamment à la hausse en raison du vieillissement démographique et des progrès technologiques, a un retentissement social et financier déjà suffisant par lui-même sans que des éléments structurels supplémentaires ne s'y ajoutent. Si des mesures devaient être prises dans ce domaine, elles devraient plutôt viser à un déplacement sur les pouvoirs publics en vue d'alléger les primes, plutôt que le contraire.

Cette situation est d'autant plus critiquable que le grand bénéficiaire de l'opération se trouve être l'assurance privée, mise au bénéfice sans aucune justification quelconque de dispositions tarifaires prévalant dans l'assurance sociale en raison de ses objectifs de santé publique, de solidarité, et de l'obligation d'assurance. Ce mélange incongru des genres n'est tout simplement pas acceptable. Même si les patients au bénéfice d'une assurance complémentaire ont légitimement droit en leur qualité de contribuables et d'assurés obligatoires aux prestations de l'Etat et de l'assurance sociale, rien ne justifie de subordonner la LCA à la LAMal et d'instaurer par la bande un frein aux dépenses dans le cadre de la prise en charge privée.

Le dispositif de planification présenté s'avère réglementé dans ses moindres détails et même compliqué sur le plan structurel, mais manifestement plutôt avare en matière de critères épidémiologiques et médicaux. Or l'un ne sera pas sans répercussion sur l'autre. Des indicateurs globaux comme le taux d'occupation ou la durée de séjour peuvent avoir des conséquences manifestement délétères sur le plan de la qualité en cas d'exigences exagérées. Dans ce contexte, les conséquences de l'application de la loi sur le travail aux assistants et chefs de clinique ne sauraient être banalisées. Le financement par le biais de prestations peut être suivi d'effets positifs sur le plan de la rationalisation souhaitable de la prise en charge dans la mesure où il reste suffisamment équilibré. En instaurant une véritable spirale du minimalisme, il peut aussi devenir pervers en récompensant ceux qui en font le moins et en pénalisant ceux qui s'engagent davantage. Tout porte à penser en outre qu'il ne se transforme rapidement en un instrument de rationnement indirect, a fortiori si les assureurs sont appelés à jouer un rôle prépondérant à terme et à exercer une pression constante sur les ressources. Cette situation serait susceptible de s'aggraver encore s'ils devaient être appelés à devenir les monistes. Ceci laisserait le soin aux services et médecins hospitaliers de limiter les prestations au gré de leurs conceptions particulières. Une telle situation n'est tout simplement pas admissible sur le plan éthique. De telles restrictions des prestations de l'assurance sociale ne sauraient être introduites par la petite porte, mais n'être décidées qu'en connaissance de cause après un très large débat démocratique. 
Le financement de la formation pré- et postgraduée ne peut pas être sans autre dissocié du financement hospitalier. La qualité des prestations et leurs résultats (outcome) en dépendent directement. Il y a donc lieu de trouver dans ce domaine une répartition équitable entre pouvoirs publics, cantons et Confédération et assureurs, qui se reporte directement sur les forfaits négociés, étant admis que les intéressés en formation y participent directement par leur travail à des conditions avantageuses.

Qu'il s'agisse de planification, du financement, ou de la qualité de la prise en charge hospitalière, une négociation partenariale équilibrée est incontournable entre autorités politiques, assureurs et fournisseurs de prestations.

\section{En particulier}

\section{Art. 22a Données de base}

Le Parlement s'est déjà exprimé à plusieurs reprises pour que les lacunes dans ce domaine soient comblées. Les modifications proposées doivent par conséquent être saluées. Mais elles ont des implications administratives et financières dont le Gouvernement semble faire totalement abstraction en postulant qu'elles sont gratuitement mises à disposition. Ces éléments doivent être pris en considération dans le financement hospitalier et la tarification ambulatoire et faire l'objet de négociations paritaires. Une attention particulière doit être apportée à la protection des données. Les données agrégées sont parfaitement suffisantes pour une appréciation de l'économicité du traitement et des procédures de prises en charge. La communication de données individuelles doit rester exceptionnelle et limitée à une demande motivée du médecin-conseil.

\section{Art. 39, al. 4 et 5}

Le mandat de prestations délivré par les cantons doit impliquer clairement une responsabilité financière de leur part. Il importe de le préciser.

La Confédération doit établir des principes généraux uniformes de planification pour toute la Suisse en accord avec les cantons, les assureurs et les fournisseurs de prestations. Les règles en la matière doivent être transparentes et les besoins clairement définis en fonction de critères épidémiologiques. Des modalités de recours doivent être envisagées. A défaut d'un dispositif décisionnel reposant sur une base légale solide, les contestations juridiques multiples sont programmées. Il y aura toujours surcapacité quelque part et un prétexte pour se dérober au financement des prestations fournies.

\section{Art. 49 , al. 3 et 4}

Comme mentionné dans les remarques générales, la formation (la recherche est un autre problème) doit être prise en considération dans le cadre du financement hospitalier.

La rémunération en cas d'hospitalisation doit faire l'objet d'une distinction claire selon qu'elle émarge uniquement à la LAMal ou également à la LCA. Les dispositions prévues par la LAMal ne s'appliquent pas à la LCA. Lorsque la LCA doit être prise en considération (assurances complémentaires), une double facturation doit être autorisée. A défaut, le système instaure clairement une médecine à 2 vitesses dans des conditions d'ambiguiité retorses particulièrement inacceptables.

\section{Dispositions transitoires II}

Il parait pour le moins incongru que les cantons participent de quelque manière que ce soit à des investissements dans le cadre d'un établissement non subventionné.

Les délais prévus par le Conseil fédéral sont peu réalistes. Il est peu probable que le système d'indemnisation forfaitaire SwissDRG débouche sur un accord entre partenaires susceptible d'entrer effectivement en vigueur avant un délai de 4-5 ans. Les conditions d'application de ce système forfaitaire sont un préalable sine qua non à toute formule moniste. Même si cette dernière justifie un profond scepticisme au gré de sa définition et de ses objectifs (un dispositif moniste dans lequel le/les assureurs seraient le/les seuls payeurs est tout simplement impensable), une réflexion à ce sujet intervenant avant même d'avoir en main les éléments nécessaires ne serait qu'un exercice de pure spéculation.

\section{Projet 2B. Managed Care}

Les objectifs primaires du Managed Care sont d'optimaliser la prise en charge des malades par la mise en place d'une démarche systématisée de type Evidence-based Medicine. Cette démarche devrait s'inscrire dans l'ensemble de la carrière médicale, des études à la pratique. Elle demande une culture médicale élargie, la mise à disposition de banques de données, et le conseil de spécialistes de la qualité. Dans le système actuel hiérarchique, ce sont les chefs de service qui procèdent au choix des meilleures approches, choix que leurs élèves répètent ensuite dans le cadre de leur pratique et de leurs responsabilités. Il s'agit de remplacer ce système de transposition du savoir par une stratégie d'élaboration plus dynamique fondée sur la concertation où le chef de 
service exerce un rôle fondamental en orientant la réflexion en fonction des diverses connaissances accumulées. Il en résulte une meilleure médecine et une meilleure efficience à la suite de choix plus approfondis. Il en résulte sans doute aussi des économies. Mais le but de cette réorientation n'est pas de faire des économies, c'est d'abord de faire de la meilleure médecine et de renforcer la concurrence sur la qualité. Un tel projet n'a strictement rien à voir avec une structure particulière d'assurance. Il n'implique aucunement une restriction du choix du médecin traitant ni d'autres contraintes.

Tout le monde devrait y être intéressé, les assurés, les patients, le corps médical et le personnel soignant, les hôpitaux, les assureurs et l'Etat. Il y aurait alors lieu de dégager les ressources nécessaires pour pouvoir encourager et développer les structures adéquates. Ce n'est malheureusement pas ce que le projet propose.

Les conditions rigides énoncées sont un véritable carcan juridico-administratif entaché d'un intolérable esprit de petit comptable. La coresponsabilité budgétaire doit faire partie du dispositif de concertation entre partenaires et non pas être une exigence préalable. Elle doit pouvoir déboucher aussi sur la mise à disposition de moyens logistiques additionnels et ne pas se borner à exercer une pression permanente, à la fin insupportable, sur les professionnels de la santé. Les rétributions par capitation ou forfaitaires comportent le danger de récompenser les prises en charge minimalistes, ce qui a manifestement quelque chose d'immoral. Ce n'est donc pas une panacée. La conception commerciale de «l'achat de prestations» spécialisées ou hospitalières à l'extérieur de l'organisation ne l'est pas davantage. Comment imaginer que les uns ou les autres puissent accorder des «avantages» à une autre organisation ou institution alors qu'ils sont déjà prisonniers de mesures tarifaires et planificatrices extrêmement contraignantes. Enfin, les assurés devraient pouvoir être récompensés pour la pertinence de leurs choix avec beaucoup plus de flexibilité. En conclusion, l'encouragement au Managed Care devrait se limiter à des mesures incitatrices. Vouloir incorporer ces aspects nouveaux de la pratique médicale dans un système d'assurance qui a ses règles propres fait manifestement fausse route. L'évolution au cours de ces dernières années du nombre d'adhérents aux HMO et autres formes particulières d'assurance proposées par les assureurs en témoigne largement. Enfin utiliser le modèle de Managed Care comme une alternative à la suppression de l'obligation/liberté de contracter est un chantage inadmissible.

\section{En particulier}

\section{Art. $41 a$, al. 1 et 2}

Le principe de la limitation du choix des fournisseurs de prestations à ceux qui sont désignés par l'assurance est une erreur de principe lourde de conséquences éthiques et sociales au départ. En témoigne le succès de plus en plus modeste de ce genre de proposition. Il y a lieu de remplacer cet article par les conditions de reconnaissance d'une organisation de Managed Care. A la limite, il devrait être biffé pour reprendre cette question dans le cadre de l'art. 41c.

\section{Art. 41b}

Plutôt que de bloquer l'assuré dans un système, on pourrait au contraire postuler qu'il pourrait bénéficier d'avantages s'il fait preuve d'une fidélité d'une durée suffisante à une organisation tout en l'autorisant à en changer s'il le désire. L'article devrait être biffé et ce genre de conditions incorporé dans un alinéa supplémentaire de l'art. 41c.

\section{Art. $41 c$, al. 2}

Cet alinéa est très problématique. Les fournisseurs de prestations conscients de leurs responsabilités de santé publique devraient pouvoir avoir accès aux données économiques les concernant et en discuter sur un plan partenarial avec les assureurs de manière à pouvoir procéder à des pesées d'intérêt et développer une optimisation des stratégies de prise en charge d'un commun accord. Mais cela n'a rien à voir avec la couverture financière des soins médicaux en tant que telle, et encore moins avec une responsabilité qui s'étend «à toutes les prestations de l'assurance obligatoire de soins». Cela n'est manifestement pas le rôle des professionnels de la santé et qui est une exigence manifestement perverse. Il est exclu de choisir une approche donnée plutôt que telle autre en fonction de critères exclusivement économiques. La question financière ne peut jouer qu'un rôle accessoire dans le choix d'approches diverses aux résultats médicaux équivalents. Il existe des banques de données pour faciliter ce genre de décisions. Ce processus devrait pouvoir bénéficier de l'assistance de spécialistes de la qualité chargés de l'évaluation des résultats sur mandat des groupes de Managed Care.

\section{Art. $41 c$, al. 3}

Evident et superflu. C'est la règle de tout processus thérapeutique, même en dehors d'une organisation de Managed Care! L'ouverture à des prestations non obligatoirement à charge des caisses-maladie est à rejeter formellement. Elle 
entre de front en contradiction avec la volonté de maîtriser les coûts qui sous-tend ce projet.

\section{Art. 41c, al. 4}

Ces questions sont l'affaire de spécialistes et ne concernent tout simplement pas le Conseil fédéral. Dès le moment où la loi en a postulé le principe, de quoi veut-il se mêler encore?

\section{Art. 52a, al. 3}

La prescription de principes actifs est une démarche positive. Contrairement aux commentaires incitant plutôt à la confusion qui accompagnent ce projet, elle témoigne dans la règle de connaissances pharmacologiques approfondies et d'une approche scientifique du problème. Les aléas liés à la préparation galénique sont un autre problème source de confusions et auquel il est attribué beaucoup trop d'importance. Il en va par contre autrement en cas de changement de marque protégée au cours d'un traitement de longue durée. Le changement d'aspect des médicaments a un effet clairement déstabilisateur au niveau de la compliance.

\section{Art. 56, al. 3bis}

On voit mal comment des avantages peuvent être reportés sur l'institution commune plutôt que sur l'assureur. Cela demande clarification.

En vous remerciant de l'attention que vous porterez à ce qui précède, veuillez recevoir, Monsieur le Conseiller fédéral, Mesdames, Messieurs, l'assurance de nos sentiments respectueux.

\section{FMH}

Dr Jacques de Haller

Président

Dr Yves Guisan

Vice-président 